TRANSACTIONS OF THE

AMERICAN MATHEMATICAL SOCIETY

Volume 252, August 1979

\title{
WIMAN-VALIRON THEORY FOR ENTIRE FUNCTIONS OF FINITE LOWER GROWTH
}

BY

P. C. FENTON

\begin{abstract}
A general method of Wiman-Valiron type for dealing with entire functions of finite lower growth is presented and used to obtain the lower-order version of a result of $\mathbf{W}$. K. Hayman on the real part of entire functions of small lower growth.
\end{abstract}

1. Introduction and statement of results. Techniques of Wiman-Valiron type for entire functions of finite upper growth have been well developed by various authors (especially [1], [2], [5], [6]) but until recently the application of these ideas to functions of finite lower growth has been hampered by the absence of a uniform method. In [3] the author applies a method-the general plan of which may be followed (at least in principle) in other cases-to functions of finite lower order. The intention here is to prove two results which make this method general-at once thereby opening a wide range of results to functions of finite lower growth-and in addition to apply them to sharpen a result due to $W$. K. Hayman.

Hayman's survey [4] begins with a fundamental result, depending on Kövari's idea of comparison functions [6], from which the subsequent applications are deduced. Let us call the negative-valued function $\alpha(t)$ a comparison function if $\alpha^{\prime}(t)<0$ for $t>0$ and $\left|\alpha^{\prime}(t)\right|$ is decreasing for $t>0$. Set

$$
\begin{gathered}
A_{n}=\exp \left(\int_{0}^{n} \alpha(t) d t\right), \quad n=0,1,2, \ldots, \\
\rho_{n}=\exp \{-\alpha(n)\}, \quad n=1,2,3, \ldots .
\end{gathered}
$$

It follows from the properties of $\alpha(t)$ that

$$
A_{n} \rho_{N}^{n}<A_{N} \rho_{N}^{N} \text { for } n \neq N \text {. }
$$

The reader is referred to the opening sections of [4] for details of this as well as other basic relations and definitions.

Given an entire function

$$
f(z)=\sum_{0}^{\infty} a_{n} z^{n}
$$

Received by the editors February 25, 1978.

AMS (MOS) subject classifications (1970). Primary 30A64. 
with central index $N(r)$, a number $r>0$ is said to be normal with respect to a comparison function $\alpha(t)$ if there exists an integer $N$ such that

$$
\frac{\left|a_{n}\right|}{\left|a_{N}\right|} r^{n-N}<\frac{A_{n}}{A_{N}} \rho_{N}^{n-N}
$$

for all $n>0$, where $A_{n}$ and $\rho_{n}$ are given by (1.1) and (1.2). If $r$ is normal then it follows from (1.3) that the integer $N$ of (1.5) is actually $N(r)$. A positive number which is not normal is called exceptional.

We have

TheOREM A ([4, TheOREM 1, p. 319]). Given the entire function (1.4), let $r_{N}$ be the smallest value of $r$ at which the central index of $f$ is $N$. Then

(i) if $\rho_{N}$ is bounded above the set of exceptional $r$ has finite logarithmic measure;

(ii) if

$$
\varlimsup_{N \rightarrow \infty} \frac{\log \rho_{N+1}}{\log r_{N}}=\delta<1,
$$

the set of exceptional $r$ has upper logarithmic density at most $\delta$;

(iii) if $\rho_{N} / r_{N} \rightarrow 0$ as $N \rightarrow \infty$ the set of normal $r$ has infinite logarithmic measure.

All results in which conclusions about density occur stem from (ii) and it is with a lower order version of this part of Theorem $A$ that we shall be concerned. We shall prove:

THEOREM 1. Suppose that $\alpha(t)$ is a comparison function which satisfies

$$
\frac{t \alpha^{\prime}(t)}{\alpha(t)} \rightarrow 0 \text { as } t \rightarrow \infty \text {. }
$$

If $f(z)$ is the entire function (1.4) and if

$$
\lim _{r \rightarrow \infty} \frac{\log \rho_{N(r)}}{\log r}=\delta<1,
$$

where $N=N(r)=N(r, f)$, then

$$
\frac{\left|a_{n}\right|}{\left|a_{N}\right|} r^{n-N}<\frac{A_{n}}{A_{N}} \rho_{N}^{n-N} \quad(0<n<2 N)
$$

outside a set $E$ of $r$ of lower logarithmic density at most $\delta$. Further, outside the same set $E$,

$$
\left|a_{n}\right|^{n}<\mu(r, f) \max \left(\left(\frac{\rho_{N}}{\rho_{2 N}}\right)^{n / 2}, \frac{A_{n}}{A_{N}} \rho_{N}^{n-N}\right) \quad(n>2 N) .
$$


The condition $0<n<2 N$ of (1.9) is arbitrary to the extent that 2 may be replaced by any $K>1$ without affecting the conclusion about $E$.

If the condition (1.7) is omitted a more limited result may be obtained which involves

$$
\lambda=\lambda(K)=\varlimsup_{n \rightarrow \infty} \frac{\alpha(K n)}{\alpha(n)}
$$

THEOREM 2. Let $\alpha(t)$ be a comparison function and let $f(z)$ be the entire function (1.4) satisfying (1.8). Given $K>1$ satisfying $0<\lambda(K)<\delta^{-1}$ we have

$$
\frac{\left|a_{n}\right|}{\left|a_{N}\right|} r^{n-N}<\frac{A_{n}}{A_{N}} \rho_{N}^{n-N} \quad(0<n<K N),
$$

where $N=N(r, f)$, outside a set $E$ of $r$ of lower logarithmic density at most $\delta /(1+\delta-\lambda \delta)$. Further, outside this same set $E$,

$$
\left|a_{n}\right| r^{n}<\mu(r, f) \max \left(\left(\frac{\rho_{N}}{\rho_{K N}}\right)^{\left(1-K^{-1}\right) n}, \frac{A_{n}}{A_{N}} \rho_{N}^{n-N}\right) \quad(n>K N) .
$$

For fixed $\delta$ and $\lambda$ such that $1<\lambda<\delta^{-1}$, the quantity $\delta /(1+\delta-\lambda \delta)$ cannot be replaced by any smaller quantity. If $\lambda>\delta^{-1}$ then all sufficiently large $r$ can be exceptional.

Finally we shall prove

THEOREM 3. Suppose that $f(z)$ is a transcendental entire function satisfying

$$
\lim _{r \rightarrow \infty} \frac{\log \log M(r, f)}{\log \log r}=p \text {. }
$$

Let

$$
B(r, f)=\max _{|z|=r} \operatorname{Re} f(z), \quad A(r, f)=\min _{|z|=r} \operatorname{Re} f(z)
$$

and define $\alpha(p)$ to be 0 if $p<2,(p-1) / p$ if $2<p<\infty, \alpha(\infty)=1$. Then given $K>1$

$$
\begin{gathered}
B(r, f)>M(r, f)\left(1-\frac{K \pi^{2} \alpha(p)+o(1)}{2 \log M(r, f)}\right), \\
-A(r, f)>M(r, f)\left(1-\frac{K \pi^{2} \alpha(p)+o(1)}{2 \log M(r, f)}\right),
\end{gathered}
$$

for $r$ in a set of upper logarithmic density at least $1-K^{-1}$.

Hayman [4, Theorem 15] obtains these beautiful inequalities subject to the stronger condition 


$$
p=\varlimsup_{r \rightarrow \infty} \frac{\log \log M(r, f)}{\log \log r},
$$

and shows that the constant $\pi^{2} \alpha(p)$ is sharp [4, Theorem 16]. The proof of Theorem 3 is thus chiefly concerned with replacing the upper limit by the lower limit.

2. Proofs of Theorems 1 and 2. The idea of the proofs is contained essentially in [3] but certain considerations of growth occurring there are in fact extraneous and by dispensing with them we are able to shorten the proofs considerably.

Let $R_{n}$ be an unbounded, increasing sequence such that

$$
\lim _{n \rightarrow \infty} \frac{\log \rho_{N}\left(R_{n}\right)}{\log R_{n}}=\delta<1,
$$

and set $M=\left[K N\left(R_{n}, f\right)\right]$, where $K=2$ for Theorem 1 and $0<\lambda(K)<\delta^{-1}$ for Theorem 2. Then

LEMMA 1. For $0 \leqslant t \leqslant R_{n} / \rho_{M}$ there exists a nonnegative integer $N_{n}=N_{n}(t)$ such that $N_{n}(t) \leqslant N\left(R_{n}\right)$ and for which

$$
\frac{\left|a_{m}\right|}{\left|a_{N_{n}}\right|} \frac{A_{N_{n}}}{A_{m}} t^{m-N_{n}} \leqslant 1 \text { for } 0 \leqslant m \leqslant M .
$$

Given any $t$ satisfying $0 \leqslant t \leqslant R_{n} / \rho_{M}$, let $q \leqslant M$ be the largest integer for which

$$
\frac{\left|a_{m}\right|}{A_{m}} t^{m} \leqslant \frac{\left|a_{q}\right|}{A_{q}} t^{q} \text { for } 0 \leqslant m \leqslant M .
$$

Then (2.2) holds with $N_{n}=q$ and we aim to show that $q<N\left(R_{n}, f\right)$.

For $N=N\left(R_{n}, f\right)<m \leqslant M$ we have, from (1.3),

$$
\begin{aligned}
\frac{\left|a_{m}\right|}{\left|a_{N}\right|} \frac{A_{N}}{A_{m}} t^{m-N} & =\frac{\left|a_{m}\right| R_{n}^{m}}{\left|A_{N}\right| R_{n}^{N}} \frac{A_{N} \rho_{m}^{N}}{A_{m} \rho_{m}^{m}}\left(\frac{t \rho_{m}}{R_{n}}\right)^{m-N} \\
& <\left(\frac{t \rho_{m}}{R_{n}}\right)^{m-N}<1 .
\end{aligned}
$$

It follows from this, together with (2.3) with $m=N$, that $q \leqslant N$ and this proves the lemma.

Concerning the numbers $N_{n}(t)$ of Lemma 1 we have:

LEMMA 2. Let $t_{n}=R_{n} / \rho_{M}$, where $M=\left[K N\left(R_{n}, f\right)\right]$. Then the numbers $N_{n}\left(t_{n}\right)$ of Lemma 1 are unbounded.

It is evidently sufficient to show that $t_{n} \rightarrow \infty$ as $n \rightarrow \infty$. Under the 
hypotheses of Theorem 1 it follows from the properties of $\alpha(t)$ that

$$
\begin{aligned}
t_{n} & =R_{n} / \rho_{M}=R_{n} \exp \left\{\alpha\left(2 N\left(R_{n}\right)\right)\right\} \\
& >R_{n} \exp \left\{\alpha\left(N\left(R_{n}\right)\right)+N\left(R_{n}\right) \alpha^{\prime}\left(N\left(R_{n}\right)\right)\right\} \\
& =\exp \left\{\log R_{n}-(1+o(1)) \log \rho_{N\left(R_{n}\right)}\right\} \\
& \rightarrow \infty \text { as } n \rightarrow \infty,
\end{aligned}
$$

from (1.7) and (2.1).

Under the hypotheses of Theorem 2

$$
\begin{aligned}
t_{n} & \geqslant R_{n} \exp \left\{\alpha\left(K N\left(R_{n}\right)\right)\right\} \\
& =R_{n} \exp \left[\alpha\left(N\left(R_{n}\right)\right) \frac{\alpha\left(K N\left(R_{n}\right)\right)}{\alpha\left(N\left(R_{n}\right)\right)}\right] \\
& \geqslant R_{n} /\left(\rho_{\left.N\left(R_{n}\right)\right)^{\lambda+o(1)}}\right. \\
& \rightarrow \infty \text { as } n \rightarrow \infty
\end{aligned}
$$

from (2.1), since $\lambda<\delta^{-1}$.

We shall prove now the inequalities (1.9), (1.10), (1.12) and (1.13) outside an exceptional set $E$, leaving the estimation of the size of $E$ to the following section.

It follows from Lemma 1 that, with $N_{n}=N_{n}(t)$ for $0 \leqslant t \leqslant t_{n}=R_{n} / \rho_{M}$,

$$
\frac{\left|a_{m}\right|}{\left|a_{N_{n}}\right|}\left(t \rho_{N_{n}}\right)^{m-N_{n}}<\frac{A_{m}}{A_{N_{n}}} \rho_{N_{n}}^{m-N_{n}} \quad\left(0 \leqslant m \leqslant K N\left(R_{n}\right)\right)
$$

and the right-hand side of (2.4) is less than one unless $m=N_{n}$. Since $N_{n} \leqslant N\left(R_{n}\right)$ and $t \rho_{N_{n}} \leqslant R_{n} \rho_{N_{n}} / \rho_{M}<R_{n}$, we have $N_{n}(t)=N\left(t \rho_{N_{n}(t)}, f\right)$ and thus (2.4) becomes

$$
\frac{\left|a_{m}\right|}{\left|a_{N}\right|} r^{m-N}<\frac{A_{m}}{A_{N}} \rho_{N}^{m-N} \quad(0<m<K N),
$$

where $N=N(r, f)$, for any $r$ in the set

$$
S=\bigcup_{n}\left\{r: r=t \rho_{N_{n}(t)} \text { and } 0 \leqslant t \leqslant t_{n}\right\} .
$$

This gives (1.9) and (1.12). Concerning (1.10) and (1.13), suppose that $r=$ $t \rho_{N_{n}(t)}, 0 \leqslant t \leqslant t_{n}$. Then, with $N_{n}=N_{n}(t)$,

$$
\left|a_{m}\right|^{m}=\left|a_{m}\right| R_{n}^{m}\left(\frac{t \rho_{N_{n}}}{R_{n}}\right)^{m} \leqslant \mu\left(R_{n}, f\right)\left(\frac{t \rho_{N_{n}}}{R_{n}}\right)^{m}
$$


and

$$
\mu\left(R_{n}, f\right)<\left(\frac{R_{n}}{t \rho_{N_{n}}}\right)^{N\left(R_{n}\right)} \mu(r, f)
$$

Also

$$
t \rho_{N_{n}}<\frac{R_{n}}{\rho_{K N\left(R_{n}\right)}} \rho_{N_{n}}<R_{n}\left(\frac{\rho_{N_{n}}}{\rho_{K N_{n}}}\right)
$$

and therefore, recalling that $N_{n}=N_{n}(t)=N(r, f)=N$, we have, for $m>$ $K N\left(R_{n}\right)$,

$$
\left|a_{m}\right|^{m}<\mu(r, f)\left(\frac{\rho_{N}}{\rho_{K N}}\right)^{m-N\left(R_{n}\right)}<\mu(r, f)\left(\frac{\rho_{N}}{\rho_{K N}}\right)^{m\left(1-K^{-1}\right)} .
$$

Finally, if $K N(r)<m<K N\left(R_{n}\right)$ then

$$
\left|a_{m}\right| r^{m}<\mu(r, f) \frac{A_{m}}{A_{N}} \rho_{N}^{m-N}
$$

and (1.10) and (1.13) are immediate.

3. Estimate for the exceptional set. The argument is standard. Precisely as in $[3$, p. 250$]$, the lower logarithmic density of the exceptional set is no more than

$$
\Delta=\underset{n \rightarrow \infty}{\lim } \frac{\log \rho_{N_{n}\left(t_{n}\right)}}{\log \left(t_{n} \rho_{N_{n}}\left(t_{n}\right)\right)}=\underset{n \rightarrow \infty}{\lim }\left(1+\frac{\log t_{n}}{\log \rho_{N_{n}\left(t_{n}\right)}}\right)^{-1} .
$$

In the case of Theorem $1, t_{n}=R_{n} / \rho_{2 N\left(R_{n}\right)}$, so

$$
\begin{aligned}
\log t_{n} & =\log R_{n}+\alpha\left(2 N\left(R_{n}\right)\right) \\
& =\log R_{n}+(1+o(1)) \alpha\left(N\left(R_{n}\right)\right) \\
& =\log R_{n}-(1+o(1)) \log \rho_{N\left(R_{n}\right)} \\
& =\log \rho_{N\left(R_{n}\right)}\left(\delta^{-1}-1+o(1)\right)
\end{aligned}
$$

from (2.1). Since $N_{n}\left(t_{n}\right)<N\left(R_{n}\right), \Delta<\delta$.

In the case of Theorem 2 we obtain

$$
\begin{aligned}
\log t_{n} & =\log R_{n}-\frac{\alpha\left(K N\left(R_{n}\right)\right)}{\alpha\left(N\left(R_{n}\right)\right)} \log \rho_{N\left(R_{n}\right)} \\
& >\log \rho_{N\left(R_{n}\right)}\left(\delta^{-1}-\lambda+o(1)\right)
\end{aligned}
$$

and this together with (3.1) gives $\Delta<\delta /(1+\delta-\lambda \delta)$. 
4. An example. Given $k>1$ and a satisfying $0<a<1$ let $g(z)$ be the entire function

$$
g(z)=\sum_{n=0}^{\infty} a_{n} z^{k^{n}}
$$

where

$$
a_{n}=\exp \left\{-k^{n(a+1)}\right\} \text {. }
$$

Given $\delta$ satisfying $0<\delta<1$ we introduce the comparison function

$$
\alpha(t)=-\delta t^{a}\left(\frac{k^{a+1}-1}{k-1}\right)
$$

Since the central index of $g$ is $k^{n}$ for

$$
k^{(n-1) a}\left(\frac{k^{a+1}-1}{k-1}\right) \leqslant \log |z|<k^{m a}\left(\frac{k^{a+1}-1}{k-1}\right)
$$

(as is readily verified) it follows that

$$
\varliminf_{R \rightarrow \infty} \frac{\log \rho_{N(R)}}{\log R}=\delta
$$

so that the hypotheses of Theorem 2 are satisfied for all $k$ and $a$ in the allowed ranges. We shall show that, given any $\varepsilon>0, \delta$ and $a$ can be found for which the exceptional set of Theorem 2 has lower logarithmic density greater than $\max \{(1-\varepsilon) \delta /(1+\delta-\lambda \delta), \delta\}$.

A short calculation yields that

$$
\frac{a_{n+1}}{a_{n}} r^{k^{n+1}-k^{n}}=\frac{A_{k^{n+1}}}{A_{k^{n}}} \rho_{k^{n}}^{k^{n+1}-k^{n}}
$$

when

$$
\log r=k^{n a}\left(\frac{k^{a+1}-1}{k-1}\right)\left(1+\delta-\frac{\delta\left(k^{a+1}-1\right)}{(a+1)(k-1)}\right)
$$

so that the logarithmic measure of the exceptional set within the interval (4.4) is

$$
-\delta k^{n a}\left(\frac{k^{a+1}-1}{k-1}\right)\left(1-\frac{k^{a+1}-1}{(a+1)(k-1)}\right) .
$$

The logarithmic measure of the exceptional set in $\left[1, r_{0}\right]$, where $r_{0}$ is the solution of (4.5) is thus

$$
-\delta\left(\frac{k^{n a}-k^{a}}{k^{a}-1}\right)\left(\frac{k^{a+1}-1}{k-1}\right)\left(1-\frac{k^{a+1}-1}{(a+1)(k-1)}\right)
$$

and the lower logarithmic density of the exceptional set is 


$$
\begin{aligned}
\Delta & =-\frac{\delta}{k^{a}-1}\left(1-\frac{k^{a+1}-1}{(a+1)(k-1)}\right)\left(1+\delta-\delta \frac{\left(k^{a+1}-1\right)}{(a+1)(k-1)}\right)^{-1} \\
& =\frac{1}{k^{a}-1}\left[\left(1+\delta-\delta \frac{\left(k^{a+1}-1\right)}{(a+1)(k-1)}\right)^{-1}-1\right] \\
& \geqslant \frac{1}{k^{a}-1}\left(\left(1+\delta-\frac{\delta k^{a}}{a+1}+\frac{\delta}{k}\right)^{-1}-1\right) \\
& =\delta\left(\frac{1}{a+1}-\frac{a}{(a+1)\left(k^{a}-1\right)}-\frac{1}{k\left(k^{a}-1\right)}\right)\left(1+\delta-\frac{\delta k^{a}}{a+1}+\frac{\delta}{k}\right)^{-1}
\end{aligned}
$$

We now allow $k$ to tend to infinity and $a$ to tend to zero in such a way that $k^{a}=\lambda$ remains constant, so that $1<\lambda<\delta^{-1}$. Then it is clear that the right-hand side of (4.6) approaches $\delta /(1+\delta-\lambda \delta)$. Thus if $\eta<\delta /(1+\delta-$ $\lambda \delta$ ) we can make $\Delta>\eta$, and this proves the last statement of Theorem 2 , when $\lambda<\delta^{-1}$.

The same example shows that, if $\lambda>\delta^{-1}$, all large values of $r$ may be exceptional. In view of (4.4) and (4.5) we see that this will be the case if for all $n$

$$
\begin{aligned}
& k^{n a}\left(\frac{k^{a+1}-1}{k-1}\right)\left(1+\delta-\delta \frac{\left(k^{a+1}-1\right)}{(a+1)(k-1)}\right) \\
&<k^{(n-1) a}\left(\frac{k^{a+1}-1}{k-1}\right)
\end{aligned}
$$

that is if

$$
\delta>\left(1-k^{-a}\right)\left(\frac{k^{a+1}-1}{(a+1)(k-1)}-1\right)^{-1} .
$$

If we set $k^{a}=\lambda$ and let $k$ tend to infinity as before, then the right-hand side of (4.8) approaches $\lambda^{-1}$. Thus if $\delta>\lambda^{-1}$ and $k$ is large enough, all values of $r$ are exceptional and the proof of Theorem 2 is complete.

5. Proof of Theorem 3: a lemma. Suppose that $f(z)$ is an entire function satisfying (1.14). Then

$$
\lim _{r \rightarrow \infty} \frac{\log N(r)}{\log \log r}=p-1,
$$

where $N(r)=N(r, f)$ is the central index of $f$ at $r$. For certainly

$$
\underline{\lim }\{\log \log \mu(r) / \log \log r\}=p
$$


and also (see $[4$, p. 318])

$$
\log \mu(r)=\int_{1}^{r} \frac{N(t)}{t} d t+O(1)<N(r) \log r+O(1),
$$

from which it follows that

$$
\varliminf_{r \rightarrow \infty} \frac{\log N(r)}{\log \log r}>p-1 .
$$

On the other hand, if there is strict inequality in (5.3) then from (5.2),

$$
\underline{\lim }\{\log \log \mu(r) / \log \log r\}>p,
$$

a contradiction, so we must have (5.1).

We shall prove:

LEMMA 3. Let $\varepsilon$ be any positive number. Then there is an increasing, unbounded sequence $R_{n}$ such that both

$$
N\left(R_{n}\right)<\left(\log R_{n}\right)^{p-1+e}
$$

and

$$
\frac{N\left(R_{n}\right)}{\log \mu\left(R_{n}\right)}<\frac{p+\varepsilon}{\log R_{n}} .
$$

We set $\log r=x, \log \mu(r)=\varphi(x)$, so that it is enough to prove that there exist arbitrarily large $x$ such that

$$
\varphi^{\prime}(x)<x^{p-1+\varepsilon}
$$

and

$$
\frac{\varphi^{\prime}(x)}{\varphi(x)}<\frac{p+\varepsilon}{x} .
$$

We note that $\varphi(x)$ is positive for $x>x_{0}$ say. Given $x_{1}>x_{0}$ we now choose $\eta$ positive but so small that

$$
\eta(p+\varepsilon)<1
$$

and

$$
\frac{\varphi(x)}{x^{p+\varepsilon}}>\eta, \quad x_{0} \leqslant x<x_{1}
$$

Since by hypothesis

$$
\varliminf_{x \rightarrow \infty} \frac{\varphi(x)}{x^{p+\varepsilon}}=0
$$

there exists a smallest $x_{2}$, where $x_{2} \geqslant x_{1}$, such that

$$
\frac{\varphi\left(x_{2}\right)}{x_{2}^{p+e}}=\eta
$$


Clearly at $x=x_{2}$,

$$
\frac{d}{d x} \log \left(\frac{\varphi(x)}{x^{p+\varepsilon}}\right)<0
$$

i.e.,

$$
\frac{\varphi^{\prime}\left(x_{2}\right)}{\varphi\left(x_{2}\right)}<\frac{p+\varepsilon}{x_{2}}
$$

which yields (5.7). Further

$$
\varphi^{\prime}\left(x_{2}\right)<\frac{p+\varepsilon}{x_{2}} \varphi\left(x_{2}\right)=(p+\varepsilon) \eta x_{2}^{p-1+\varepsilon}<x_{2}^{p-1+\varepsilon}
$$

which yields (5.6).

6. Hayman's inequality. We may assume that $p<\infty$, since the case $p=\infty$ was proved by Hayman [4], and we consider first the case $2<p<\infty$. Let $\alpha(t)$ be the comparison function

$$
\alpha(t)=-t^{1 /(p-1+\eta)},
$$

where $\eta$ is a positive number, and let $R_{n}$ be the sequence of Lemma 3 corresponding to $\varepsilon$, where $0<\varepsilon<\eta$. Then (2.1) holds with $\delta=0$. We conclude from (the proof of) Theorem 2 that the inequalities (1.12) and (1.13) hold, with $K=2$ (say), for $r$ in $\left[1, t_{n} \rho_{N_{n}\left(t_{n}\right)}\right]$ outside a set of logarithmic measure $o\left(\log \left(t_{n} \rho_{N_{n}\left(t_{n}\right)}\right)\right)$. However $t_{n} \rho_{N_{n}\left(t_{n}\right)}<R_{n}$ and also

$$
\begin{aligned}
t_{n} \rho_{N_{n}\left(t_{n}\right)} & >t_{n}=R_{n} / \rho_{2 N\left(R_{n}\right)} \\
& =R_{n} \exp \left(-\left(2 N\left(R_{n}\right)\right)^{1 /(p-1+\eta)}\right)=R_{n}^{1+o(1)}
\end{aligned}
$$

from (2.1) with $\delta=0$. It follows that $t_{n} \rho_{N_{n}\left(t_{n}\right)}=R_{n}^{1+o(1)}$ and therefore (1.12) and (1.13) hold, with $K=2$, in $\left[1, R_{n}\right]$ outside a subset of logarithmic measure $\varepsilon\left(R_{n}\right) \log R_{n}$, where $\varepsilon\left(R_{n}\right) \rightarrow 0$ as $n \rightarrow \infty$.

We quote the following result, the proof of which is virtually identical to that of the corresponding result in [4, p. 338], depending only on Theorem 2 . The single modification needed is the separate incorporation of (1.13) as an estimate for the tail terms of the Taylor series of $f$ but since (1.13) leads to a better estimate than the one already dealt with in [4] the change in the proof is minimal.

LEMMA 4. Suppose that $f(z)$ satisfies (1.14) and that $r$ is a number at which (1.12) and (1.13) hold. If $z_{0},\left|z_{0}\right|=r$, is such that

$$
f\left(z_{0}\right)=M(r) e^{i \lambda},
$$

where $\lambda$ is real, then

$$
\log f\left(z_{0} e^{i \theta}\right)=\log f\left(z_{0}\right)+i a(r) \theta-\varphi_{2} \theta^{2}+\delta(\theta)
$$


for $-\frac{1}{30} k<\theta<\frac{1}{30} k$, where $a(r)=r(d / d r) \log M(r)$ and

$$
k=\left[10 N^{(p-2+\eta) /(p-1+\eta)} \log N\right]^{1 / 2}
$$

Moreover $\left|\varphi_{2}\right|<\frac{1}{2} b(r)$, where $b(r)=(r d / d r)^{2} \log M(r)$, and $|\delta(\theta)|<$ $4(18 k|\theta|)^{3}$.

Following Hayman [4, p. 350] we obtain from this

$$
B(r) \geqslant M(r)\left[1-\frac{(1+o(1)) \pi^{2} b(r)}{2 a(r)^{2}}+O\left(k^{3} a(r)^{-3}\right)\right]
$$

for all $r$ for which (1.12) and (1.13) hold. Since $\log M(r)$ is an increasing convex function of $\log r$ which satisfies (1.14) we deduce that, for all $r$,

$$
\log M(r)=O\{a(r) \log r\}=O\left\{a(r)^{p /(p-1)+o(1)}\right\}
$$

and since $N(r) \sim a(r)$ as $r \rightarrow \infty$ through values for which (1.12) and (1.13) hold (see [4, pp. 352, 353]) we readily obtain

$$
k^{3} a(r)^{-3}=o(\log M(r))^{-1}
$$

and therefore

$$
B(r)>M(r)\left[1-\frac{(1+o(1)) \pi^{2} b(r)}{2 a(r)^{2}}+o(\log M(r))^{-1}\right]
$$

for all $r$ in $\left[1, R_{n}\right]$ outside a subset of logarithmic measure $\varepsilon\left(R_{n}\right) \log R_{n}$. It remains now only to estimate $b(r) / a(r)^{2}$ which we do by means of (5.5) together with Lemma 9 of $[4$, p. 351].

We select a normal value of $r$, say $r_{n}$, from $\left[R_{n}^{1-2 e\left(R_{n}\right)}, R_{n}\right]$. Then

$$
\log \mu\left(R_{n}\right)-\log \mu\left(r_{n}\right)=\int_{r_{n}}^{R_{n}} \frac{N(t)}{t} d t<2 N\left(R_{n}\right) \varepsilon\left(R_{n}\right) \log R_{n}
$$

so that, from (5.5),

$$
\frac{N\left(r_{n}\right)}{\log \mu\left(r_{n}\right)}<\frac{N\left(R_{n}\right)}{\log \mu\left(r_{n}\right)}<\frac{N\left(R_{n}\right)}{\log \mu\left(R_{n}\right)-2 N\left(R_{n}\right) \varepsilon\left(R_{n}\right) \log R_{n}}<\frac{p+\varepsilon^{\prime}}{\log r_{n}}
$$

for all large $n$, where $\varepsilon^{\prime}>\varepsilon$, the number of Lemma 3. Since $r_{n}$ is normal we have

$$
\frac{a\left(r_{n}\right)}{\log M\left(r_{n}\right)}<\frac{a\left(r_{n}\right)}{\log \mu\left(r_{n}\right)}=\frac{(1+o(1)) N\left(r_{n}\right)}{\log \mu\left(r_{n}\right)}<\frac{p+\varepsilon^{\prime \prime}}{\log r_{n}}
$$

for all large $n$, where $\varepsilon^{\prime \prime}>\varepsilon^{\prime}$. It follows from (6.5) (see [4, p. 351]) that

$$
\frac{b(r) \log M(r)}{a(r)^{2}} \leqslant K\left(1-\left(p+\varepsilon^{\prime \prime}\right)^{-1}\right)
$$


for all $r$ in $\left[1, r_{n}\right]$ outside a subset of logarithmic measure no more than $K^{-1}$. The set of normal values in $\left[1, r_{n}\right]$ for which (6.6) holds thus has logarithmic measure at least $\left(1-K^{-1}+o(1)\right) \log r_{n}$ and from this together with (6.4) we deduce that for these normal values

$$
B(r) \geqslant M(r)\left[1-\frac{(1+o(1)) K \pi^{2}\left(1-\left(p+\varepsilon^{\prime \prime}\right)^{-1}\right)}{2 \log M(r)}\right] .
$$

From (6.7) we obtain by a standard argument-constructing a new sequence from the various sequences $\left(r_{n}\right)$ corresponding to arbitrarily small values of $\varepsilon^{\prime}$ (and so of $\varepsilon$ )-the inequality (1.15). The second part follows from the consideration of $-f(z)$.

There remains only the case $p<2$. The proof follows exactly that of the corresponding case in [4], except that Hayman's Lemma 4 is replaced by its lower order analogue. The proof of the latter is effected by straightforward modifications.

ACKNOWLEDGEMENTS. The author is indebted to the referee for several valuable suggestions. The proof of Lemma 3, which replaces the clumsy original of more than three times the length, is his and parts of the proof of Theorem 2 have been clarified as a result of his remarks.

\section{REFERENCES}

1. J. Clunie, The determination of an integral function of finite order by its Taylor series, J. London Math. Soc. 28 (1953), 58-66.

2. On the determination of an integral function from its Taylor series, J. London Math. Soc. 30 (1955), 32-42.

3. P. C. Fenton, Some results of Wiman-Valiron type for integral functions of finite lower order, Ann. of Math. (2) 103 (1976), 237-252.

4. W. K. Hayman, The local growth of power series: a survey of the Wiman-Valiron method, Canad. Math. Bull. 17 (1974), 317-358.

5. T. Kövari, On theorems of G. Pólya and P. Turan, J. Analyse Math. 6 (1958), 323-332.

6. On the Borel exceptional values of lacunary integral functions, J. Analyse Math. 9 (1961), 71-109.

Department of Mathematics, University of Otago, Dunedin, New Zealand 